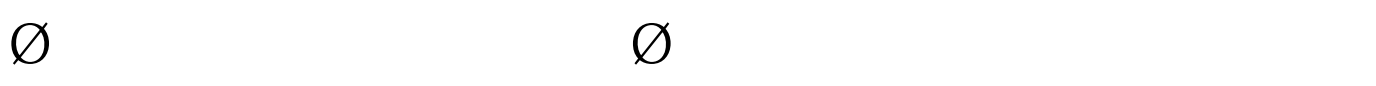

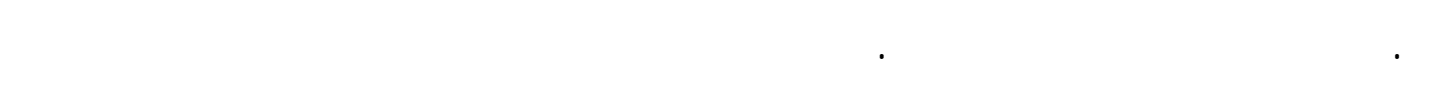

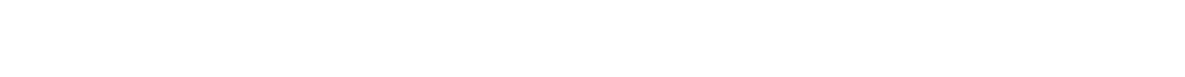

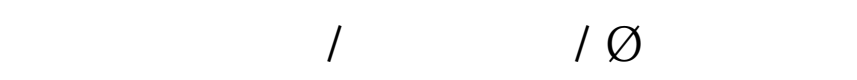

\begin{abstract}
ŭ ŤśE

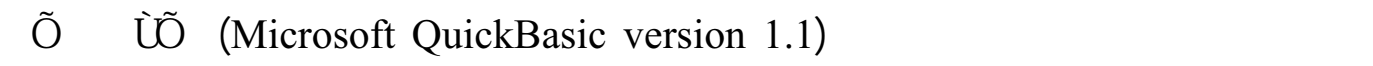

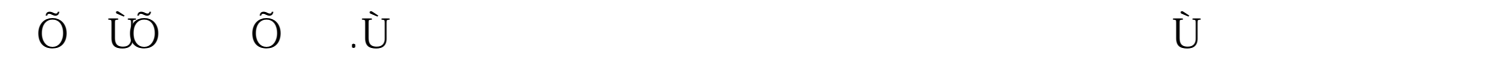

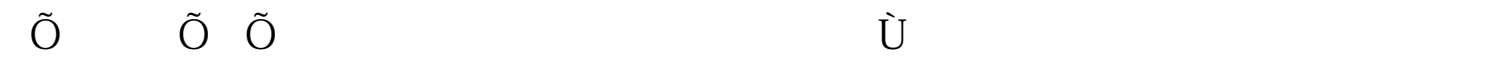

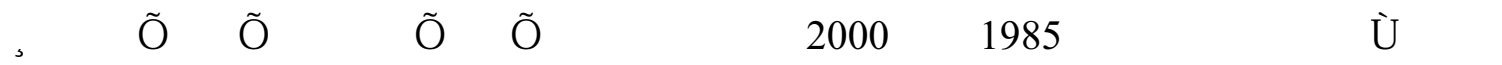

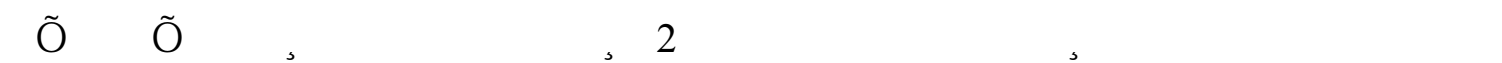

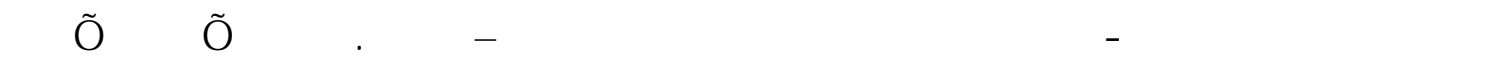

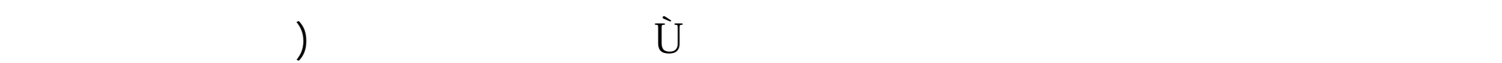

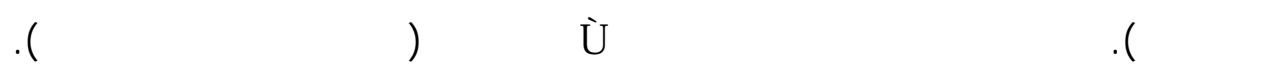

\section{Modeling Deficit Irrigation Water Requirement For Maize in Mosul Region}

Dr.Anmar A.AL-Talib Dr. Abdul Sattar Y.AL-Dabagh Ahmed A.AL-Neami

Ass. Professor

Professor

M.Sc.

Water Resources Department/ College of Engineering/ University of Mosul

\begin{abstract}
A computerized model with (Microsoft QuickBasic version 1.1) was proposed for simulating the effect of deficit irrigation for maize crop during spring and autumn seasons in Mosul region. The simulation is based on 16 years of climatological data for the period ( 1985-2000) for Mosul meteorological station, which includes daily maximum and minimum temperatures, maximum and minimum relative humidity , wind speed at $2 \mathrm{~m}$ height, and sunshine hours, which is used to calculate daily reference evapotranspiration with Penman-Monteith equation .The model predicts yield reduction by changing irrigation depth for three different irrigation methods (sprinkler, furrow and drip) .The rainfall is divided into three classes which represent three regions (wet ,semi- arid and arid ).
\end{abstract}




\section{ํㅜㄴㅗำ}

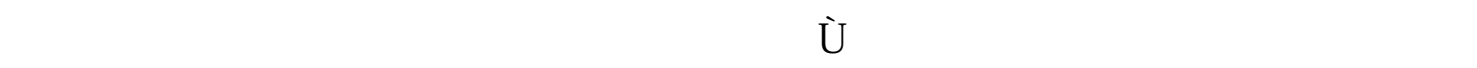

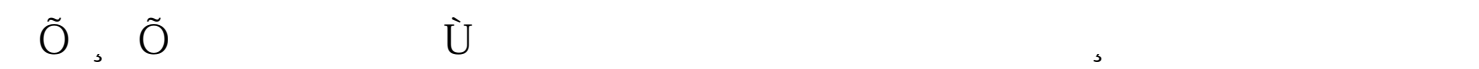

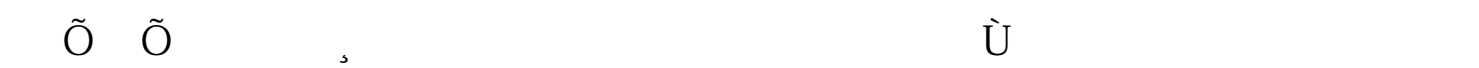

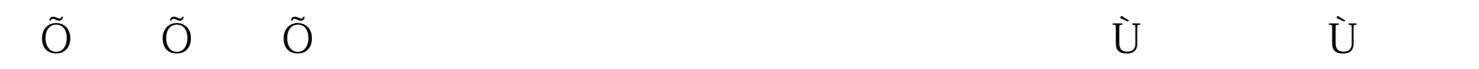

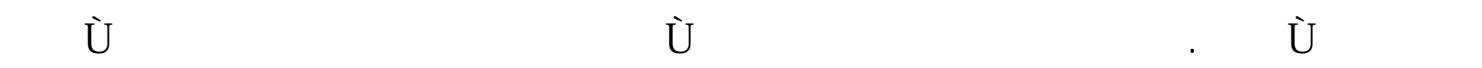

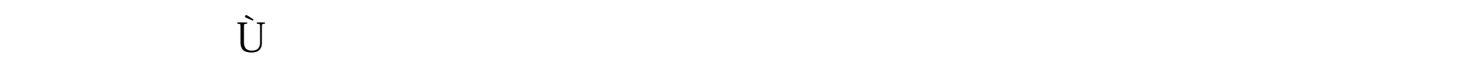

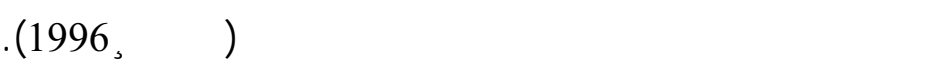

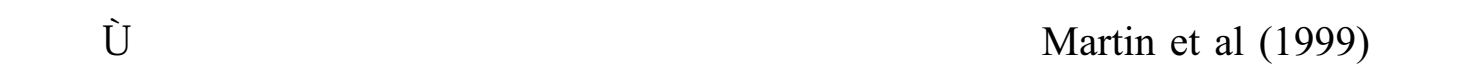

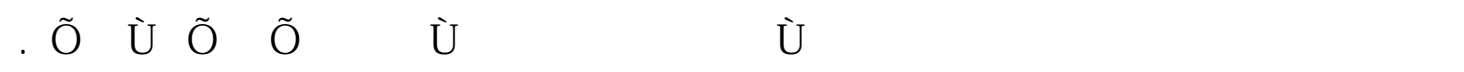

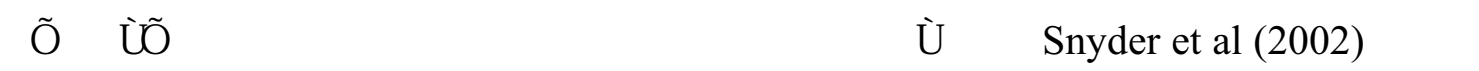
- ¿ 再 も»

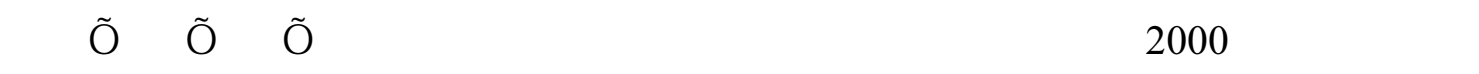

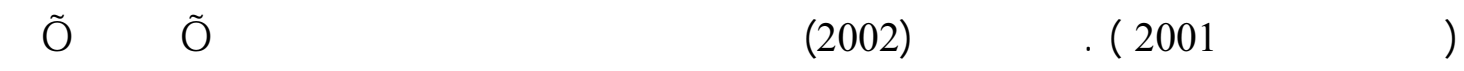
Đ) C Śr

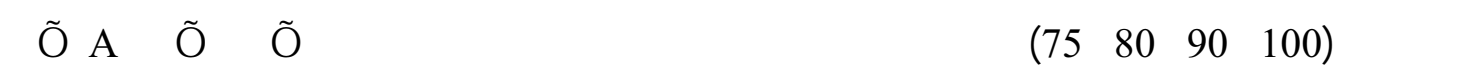

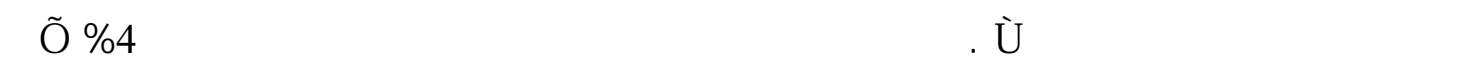

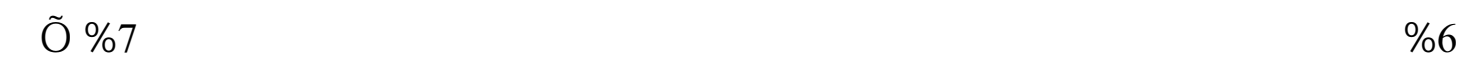

$\% 11$

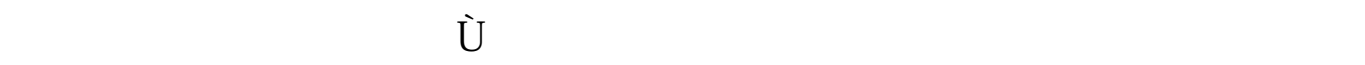

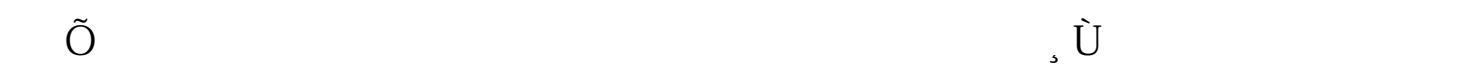

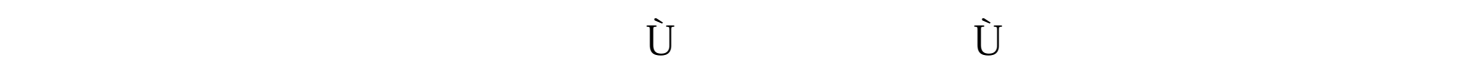

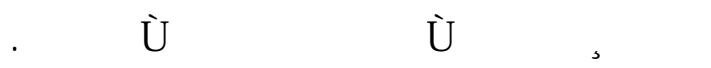

\section{Śšntrịt:ü}

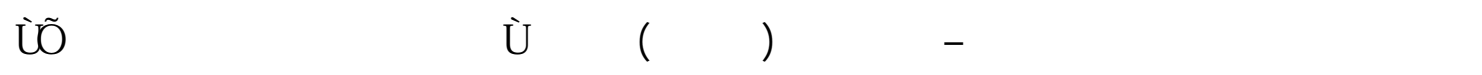

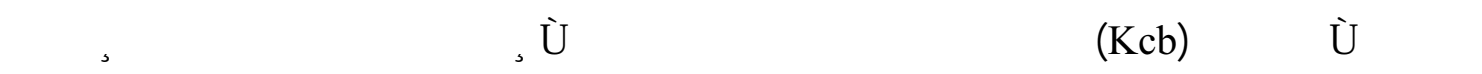

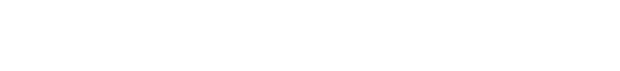

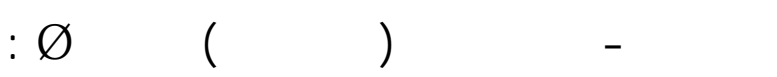

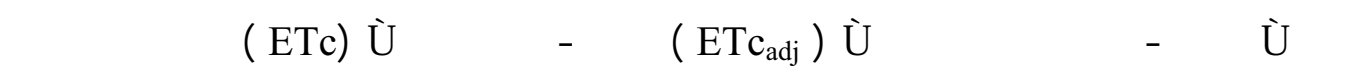

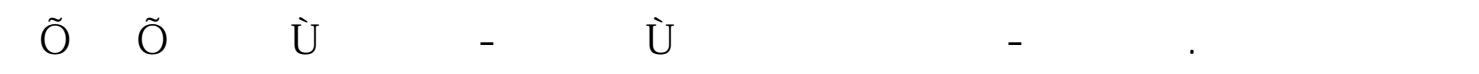

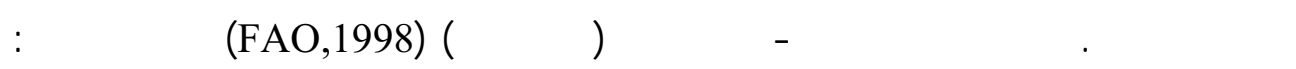


$\mathrm{ETc}_{\mathrm{adj}}=(\mathrm{Ks} * \mathrm{Kcb}+\mathrm{Ke}) \mathrm{ETo}$

$: \boxminus \overline{a ̄ e}$

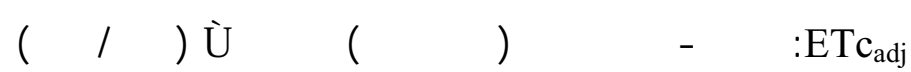

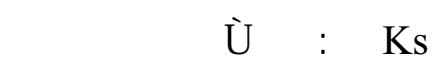

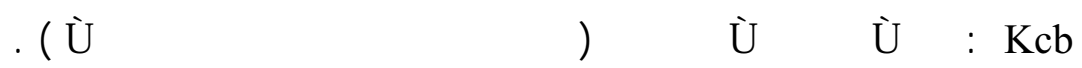

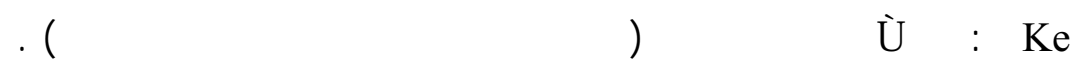

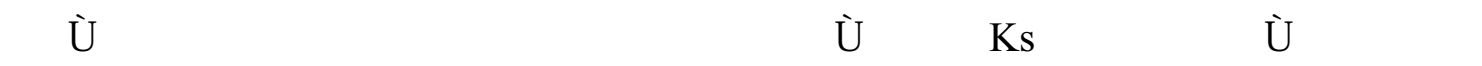

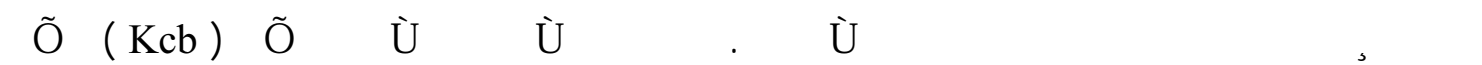

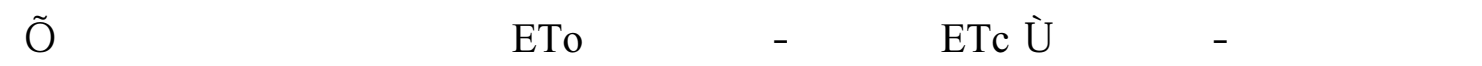

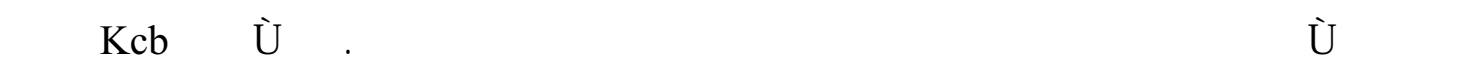

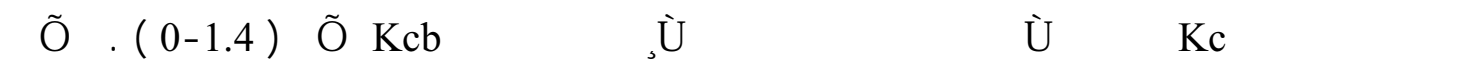

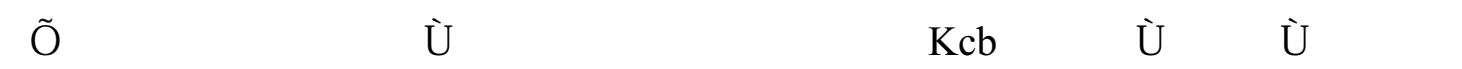

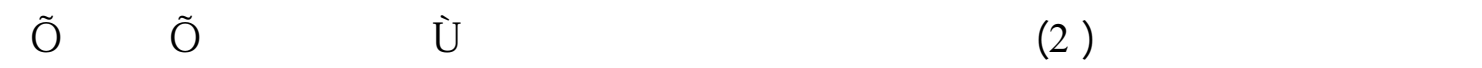

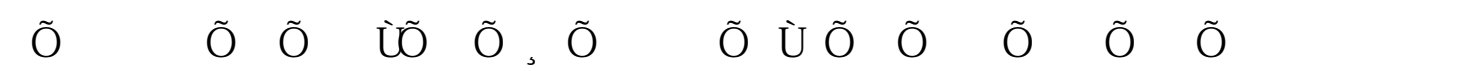

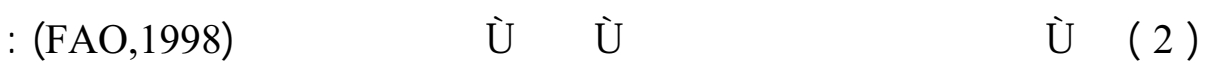
$\mathrm{Kcb}_{\mathrm{i}}=\mathrm{Kcb}_{\text {prev }}+\left[\frac{\mathrm{i}-\sum\left(\mathrm{L}_{\text {prev }}\right)}{\mathrm{L}_{\text {stage }}}\right]\left[\mathrm{Kcb}_{\text {next }}-\mathrm{Kcb}_{\text {prev }}\right]$

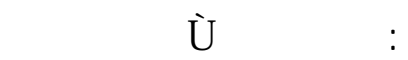
:Đẫũ

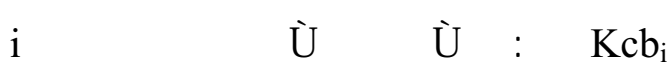

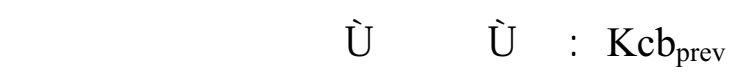

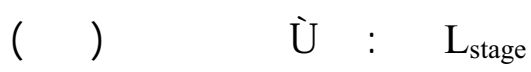

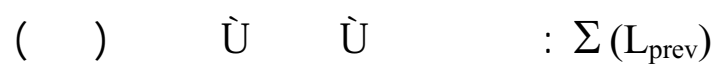

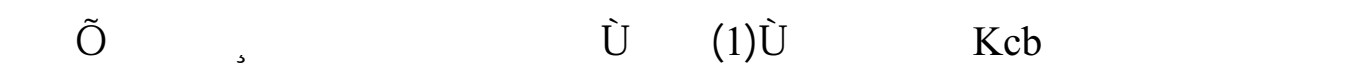

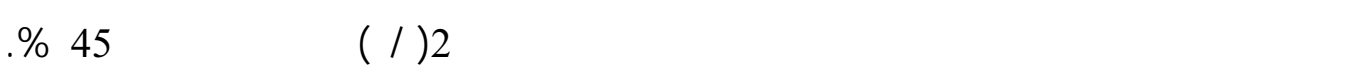

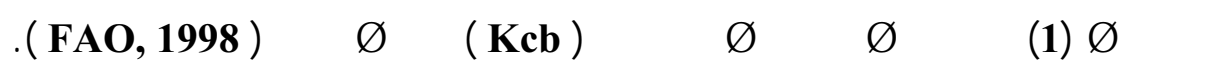

\begin{tabular}{|c|c|c|c|c|}
\hline 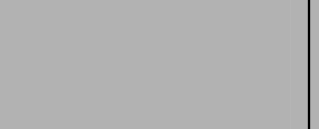 & 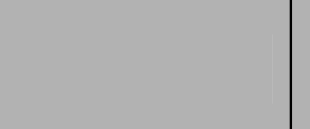 & 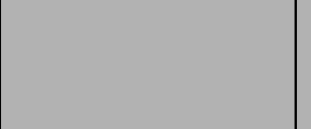 & 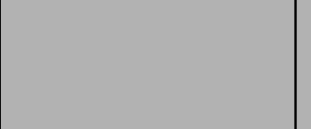 & 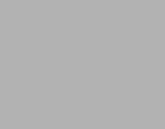 \\
\hline 0.15 & 1.15 & - & 0.15 & If́fítrE \\
\hline
\end{tabular}




\begin{tabular}{|c|c|c|c|c|}
\hline 0.5 & 1.15 & $\longleftarrow$ & 0.15 & 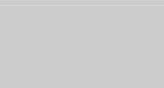 \\
\hline
\end{tabular}

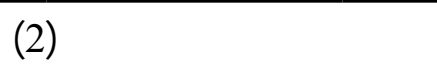

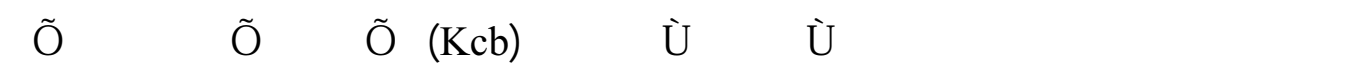

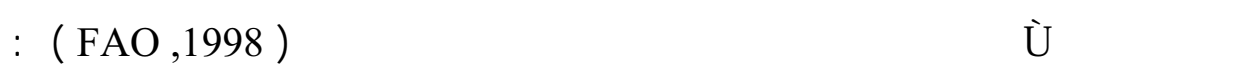
$\mathrm{Kcb}=\mathrm{Kcb}_{(1 \mathrm{~J})}+\left[\begin{array}{lll}0.04(\mathrm{U} 2-2) & -0.004\left(\mathrm{RH}_{\min }-45\right)\end{array}\right]\left(\frac{\mathrm{h}}{3}\right)^{0.3}$

:Đẫ̃

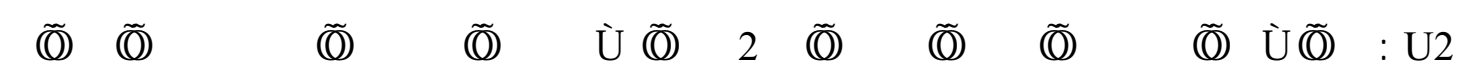

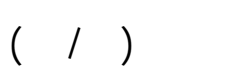

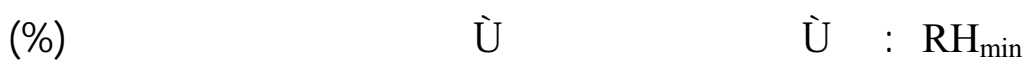
Đ»» » »C ř (C) วथर्मर

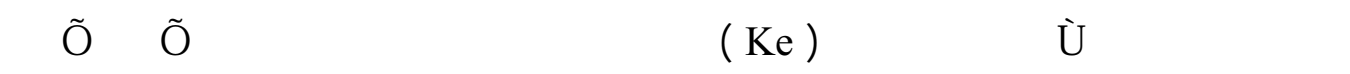

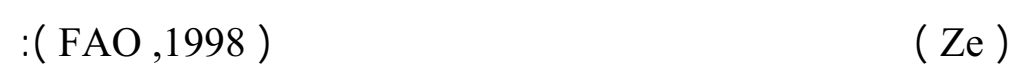
$\mathrm{Ke}=\min \left(\mathrm{Kr}\left(\mathrm{Kc}_{\max }-\mathrm{Kcb}\right)\right.$, few $\left.* \mathrm{Kc}_{\text {max }}\right)$ $: \boxminus \bar{a} \tilde{\mathbb{B}}$

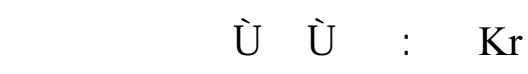

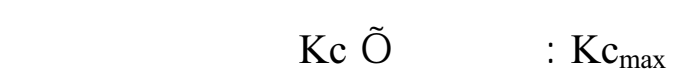

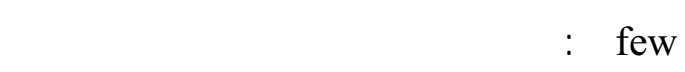

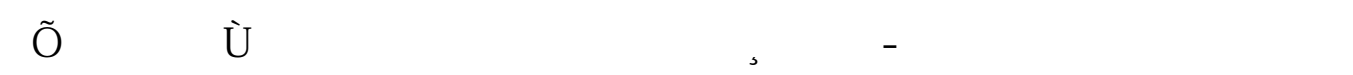

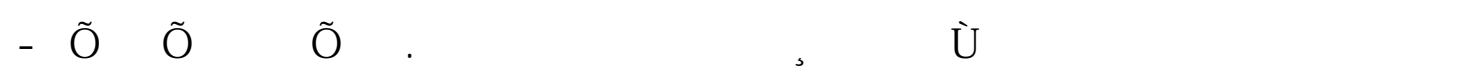

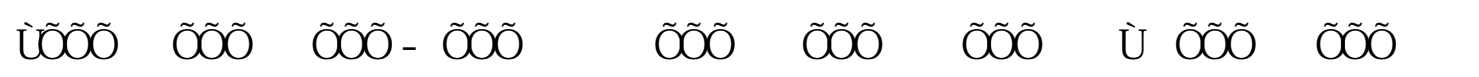
.( Sumner,1996)

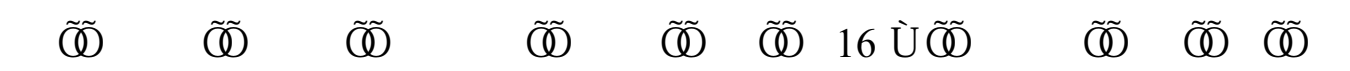

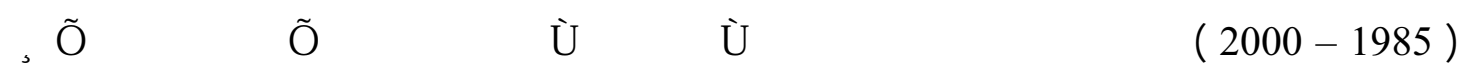

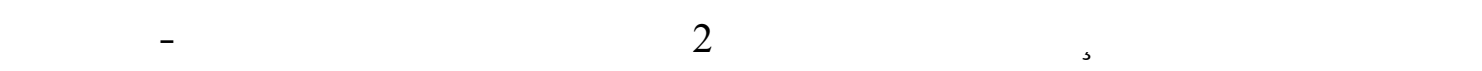

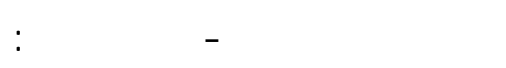

$\mathrm{ETo}=\frac{0.408 \Delta(\mathrm{Rn}-\mathrm{G})+\gamma \frac{900}{(\mathrm{~T}+273)} \mathrm{U} 2(\mathrm{es}-\mathrm{ea})}{\Delta+\gamma(1+0.34 \mathrm{U} 2)}$

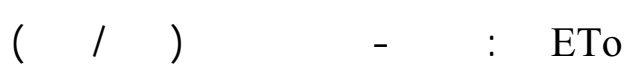

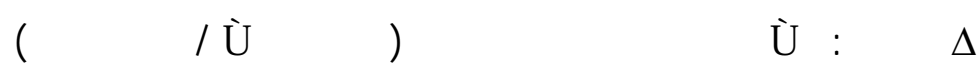

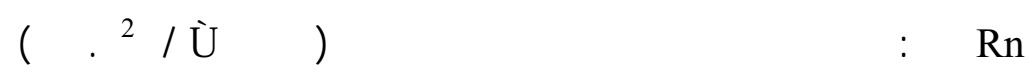




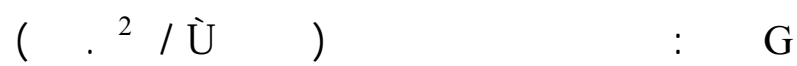

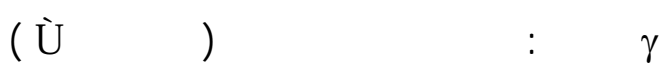

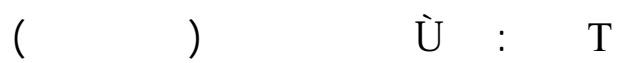

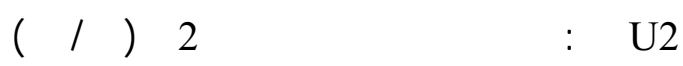

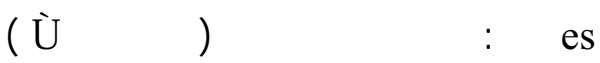

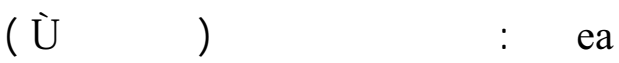

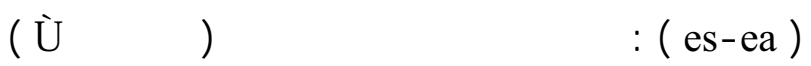

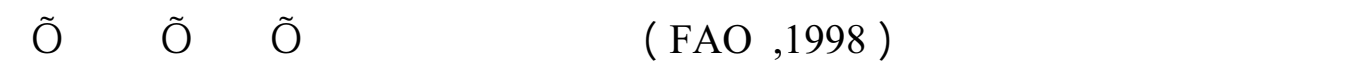

. Ŝ Đít

\section{: Ŝč̛ớă toúr}

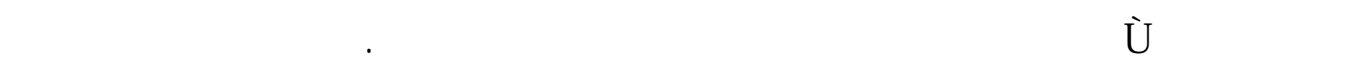

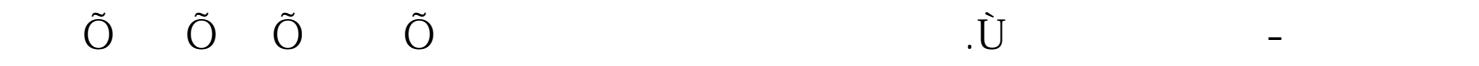

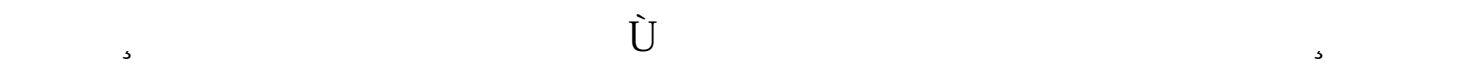

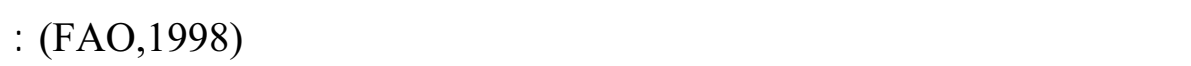

$$
\left(1-\frac{\mathrm{ya}}{\mathrm{ym}}\right)=\mathrm{Ky}\left(1-\frac{\mathrm{ETc}_{\mathrm{adj}}}{\mathrm{ETc}}\right)
$$

$: \boxminus \bar{a} \tilde{\mathbb{R}}$

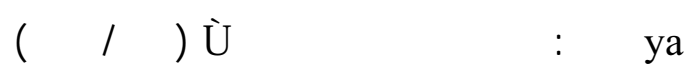

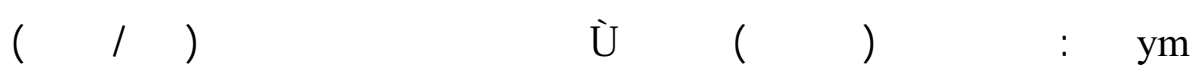

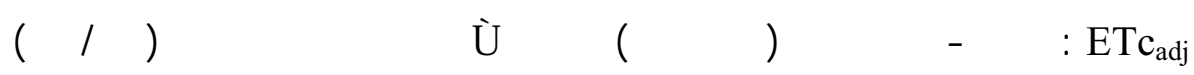

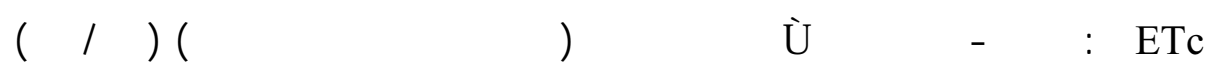

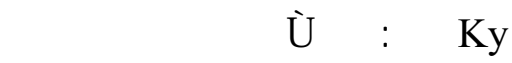

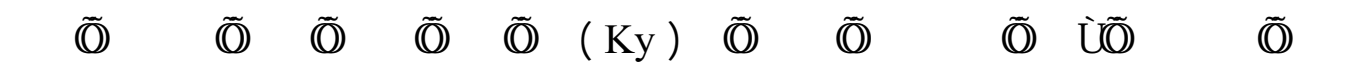

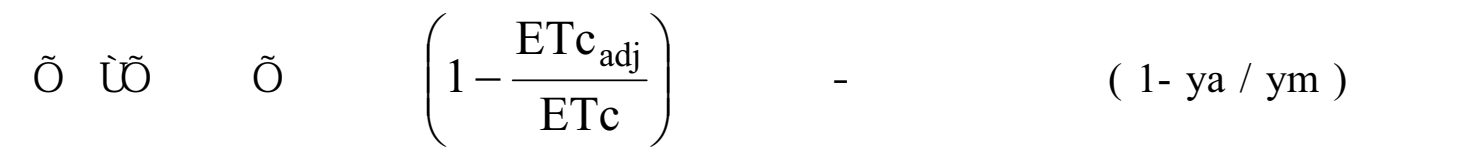
¿» Ščs

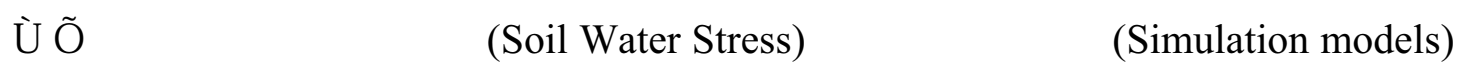

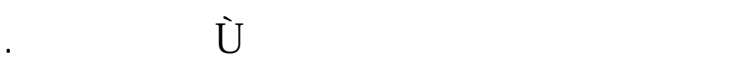

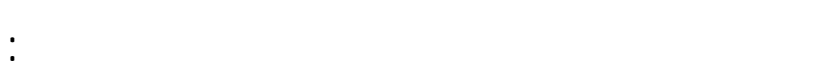

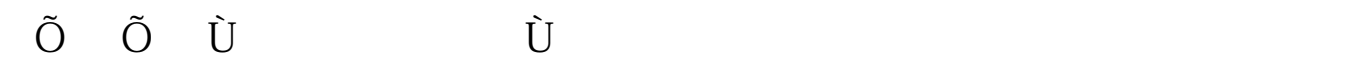

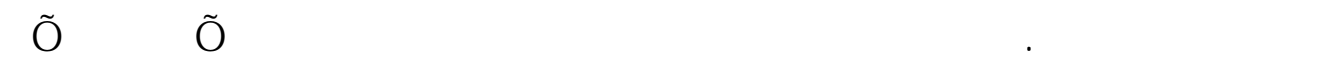

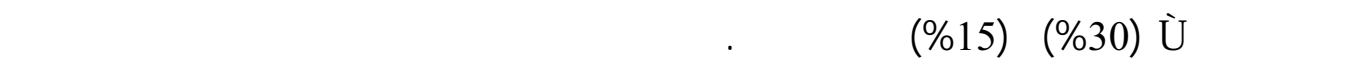




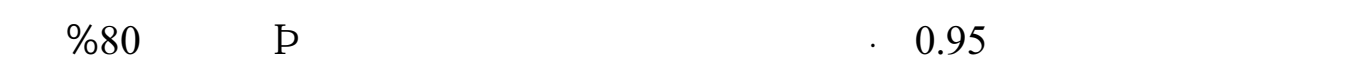

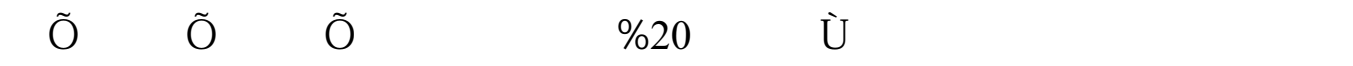

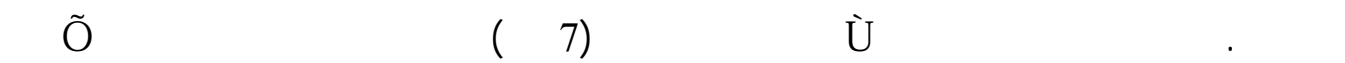

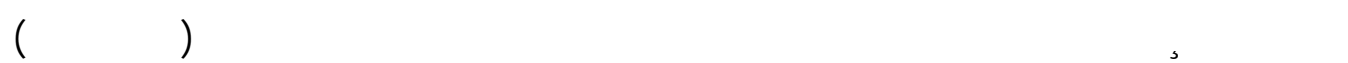

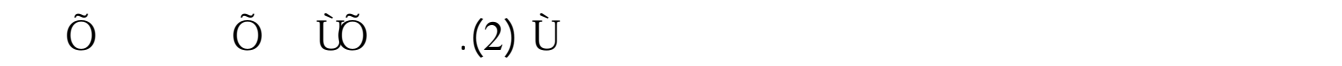

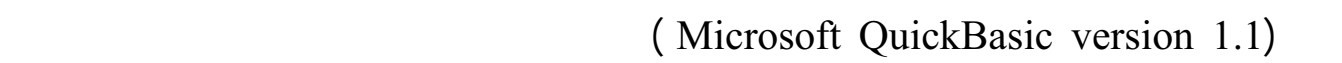

: rosḱ

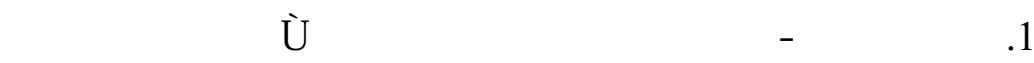

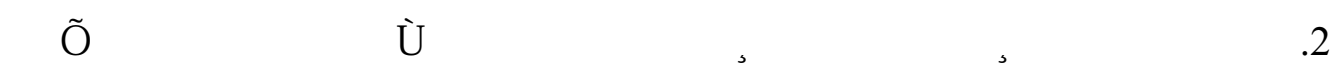

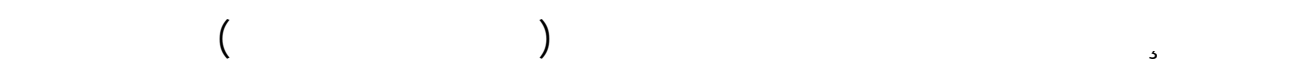

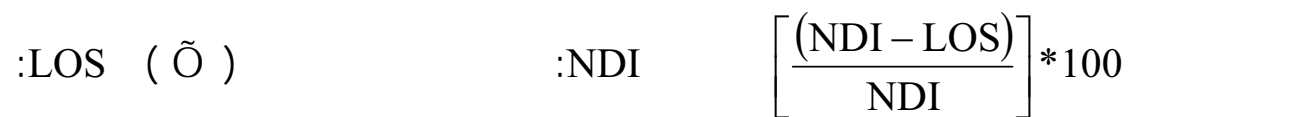

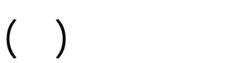

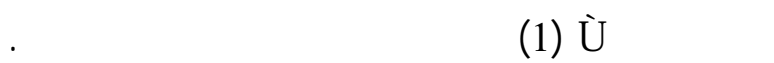

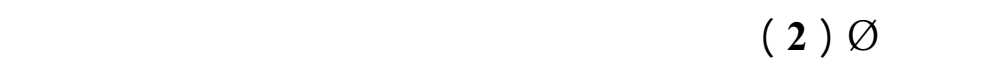

\begin{tabular}{|c|c|c|c|c|c|}
\hline 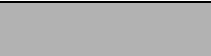 & \multicolumn{3}{|c|}{ 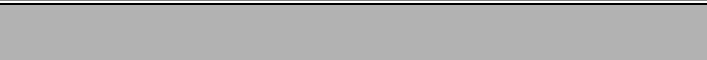 } & \multirow{2}{*}{ 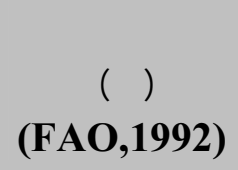 } & \multirow{2}{*}{$\varepsilon$ Elorftüt } \\
\hline ( & 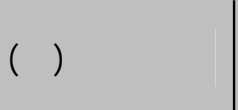 & 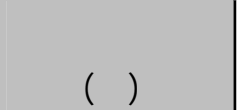 & 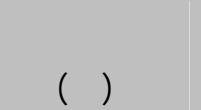 & & \\
\hline 30 & 30 & 20 & 10 & $10-30$ & üforthe \\
\hline 60 & 60 & 45 & 30 & $30-60$ & ŬbEXIE \\
\hline 80 & 80 & 55 & 30 & $30-80$ & ū tFIE \\
\hline
\end{tabular}

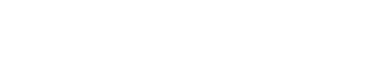

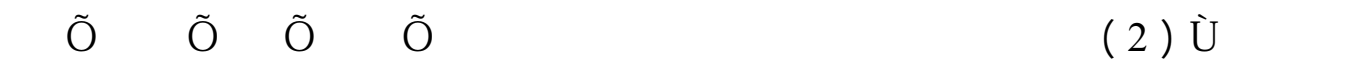

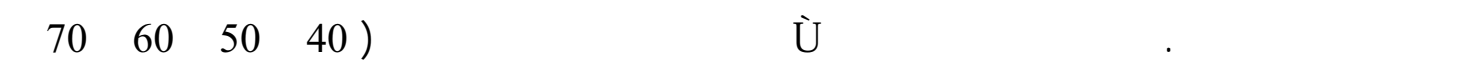

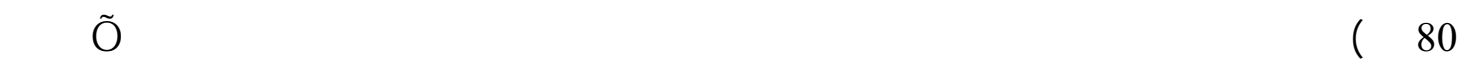

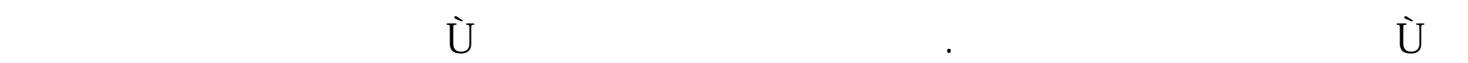

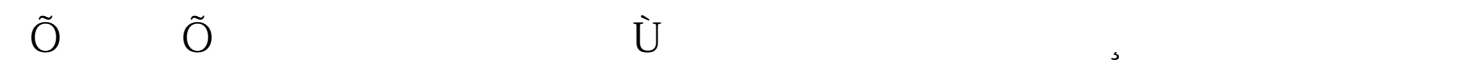

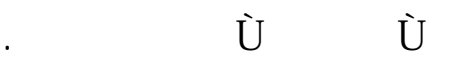

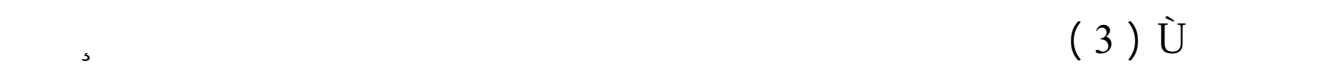

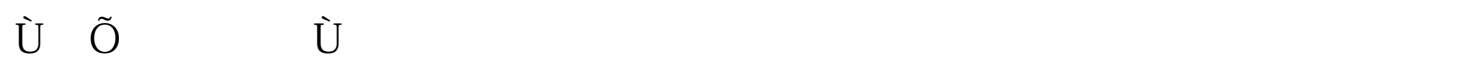
¿" E

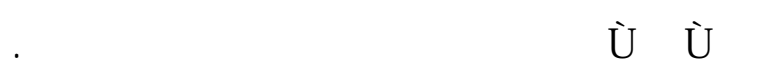

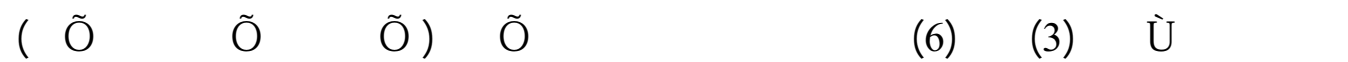

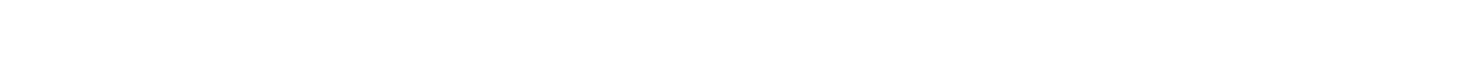




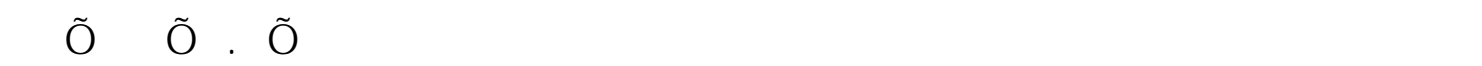

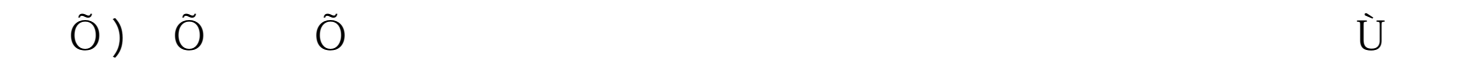

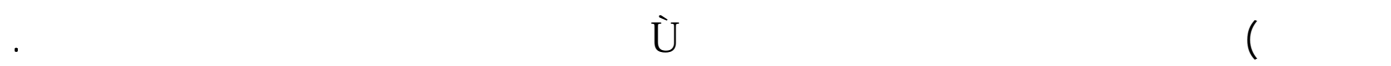

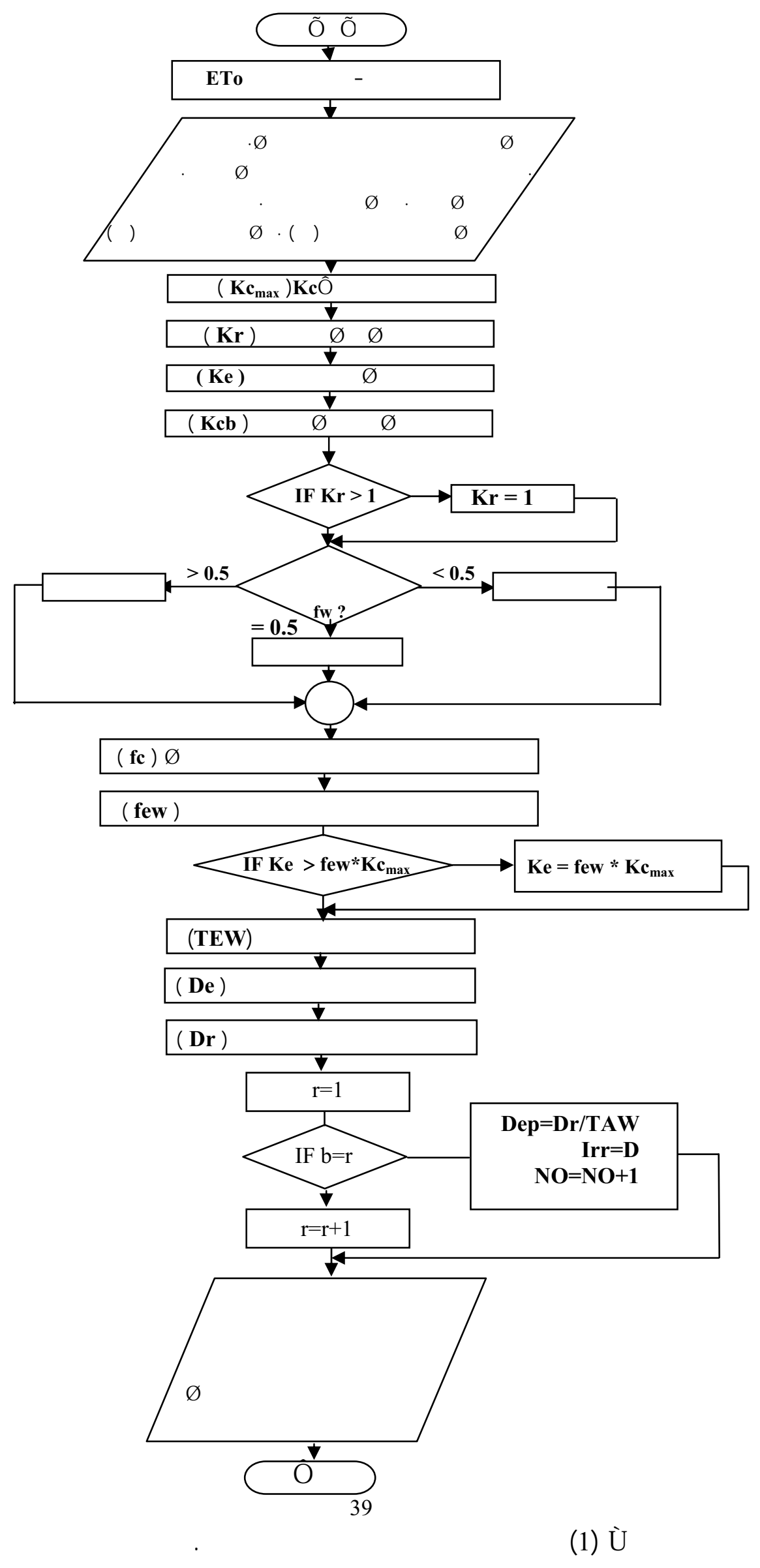




\section{ŘçǒłứKG}

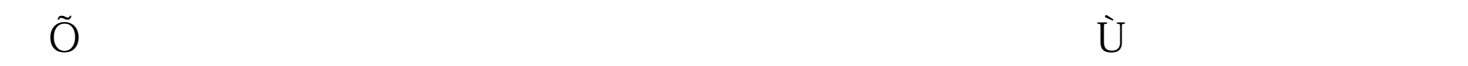

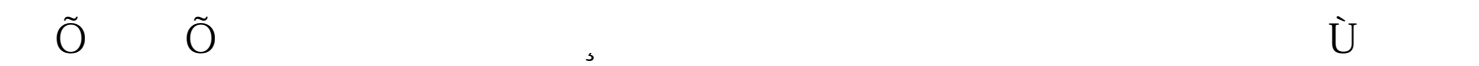

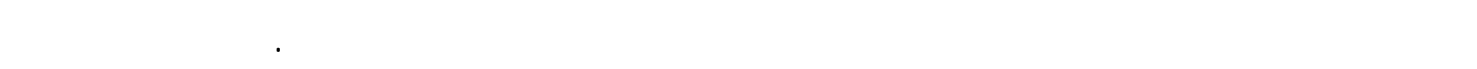

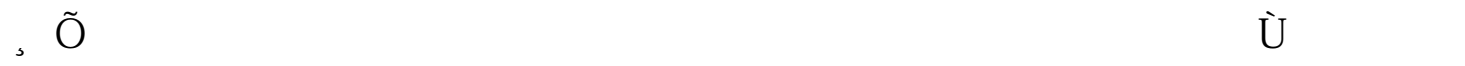

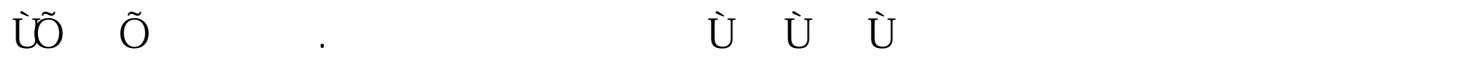

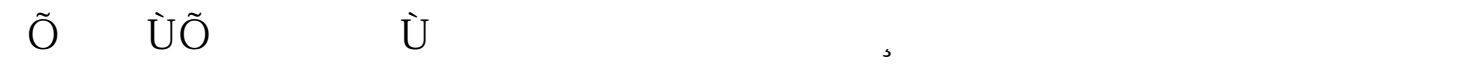

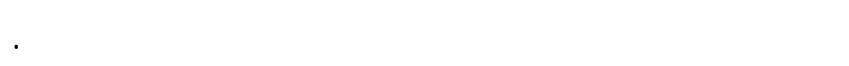

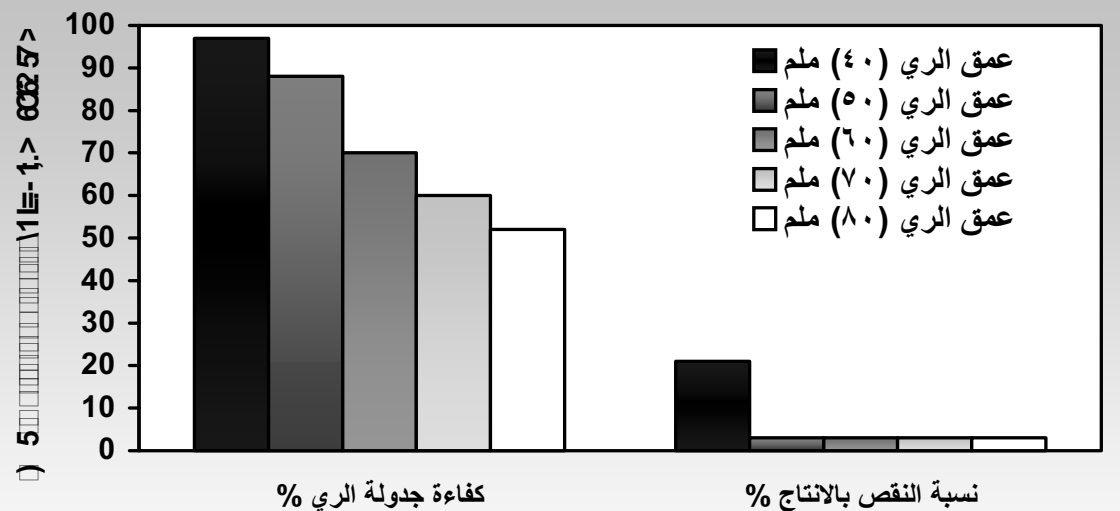

पा

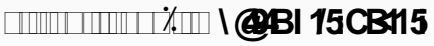

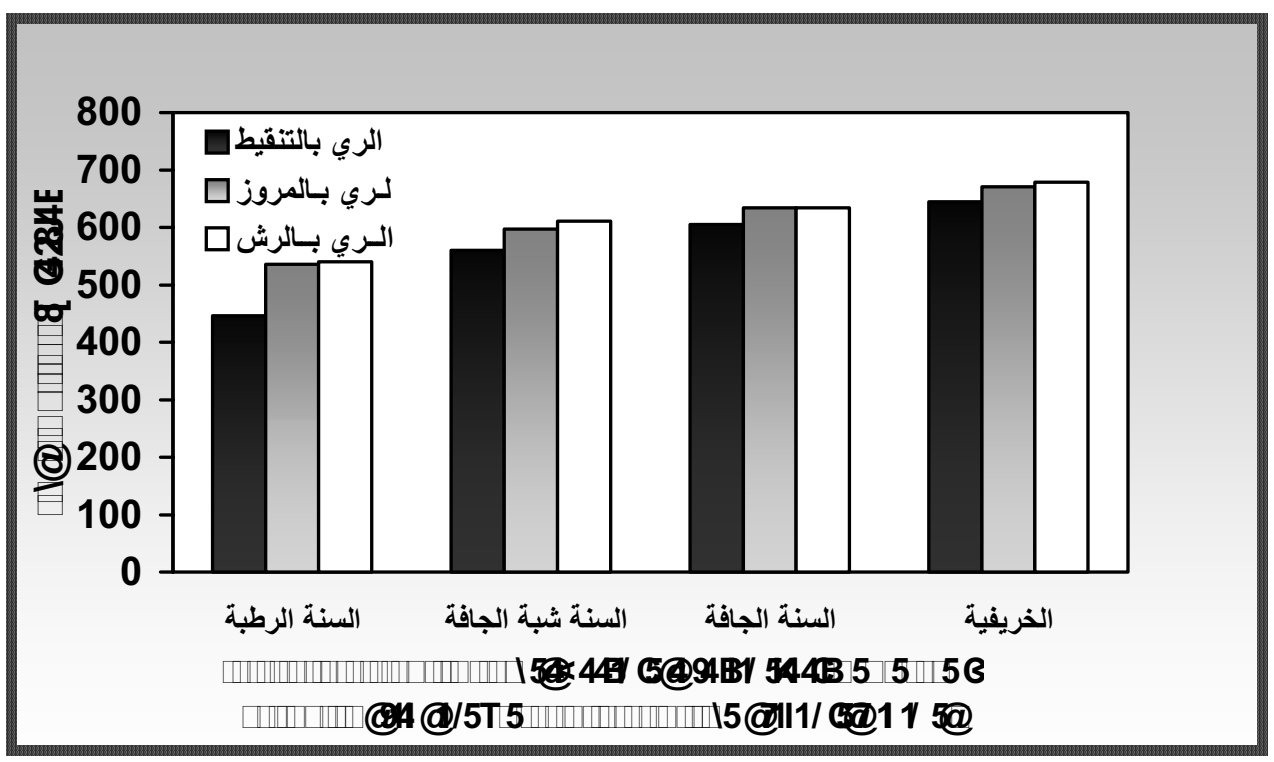




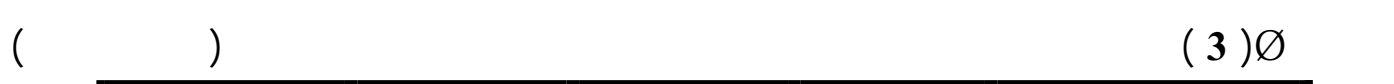

\begin{tabular}{|c|c|c|c|c|}
\hline 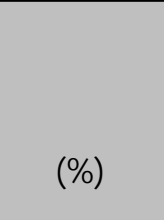 & 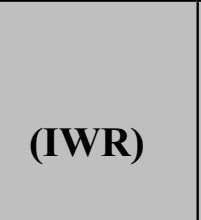 & 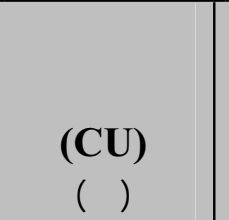 & 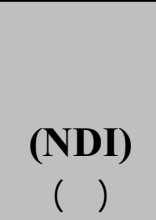 & $\varepsilon$ Etriftü \\
\hline 17 & 446 & 495 & 170 & úfiartióet|rie \\
\hline 2 & 536 & 608 & 510 & 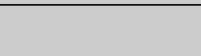 \\
\hline 2 & 540 & 618 & 510 & ū \\
\hline
\end{tabular}

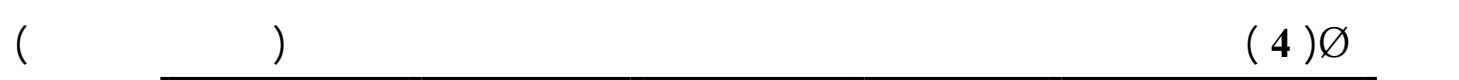

\begin{tabular}{|c|c|c|c|c|}
\hline 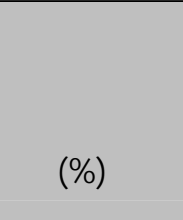 & 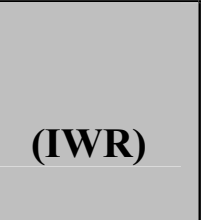 & 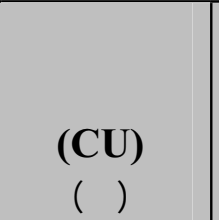 & 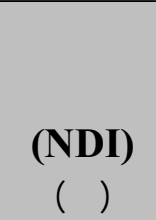 & $\varepsilon \neq 1$ (1) \\
\hline 31 & 560 & 480 & 340 & úfurfoetrie \\
\hline .6 & 597 & 648 & 765 & 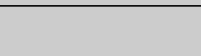 \\
\hline .2 & 611 & 670 & 935 & ū tlócet邽 \\
\hline
\end{tabular}

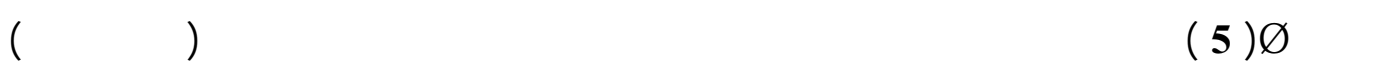

\begin{tabular}{|c|c|c|c|c|}
\hline 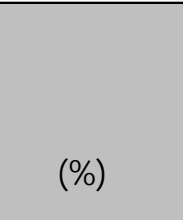 & 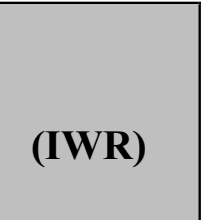 & 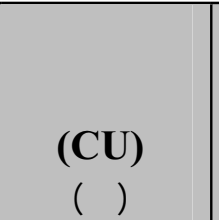 & 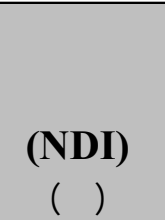 & Etroftétú \\
\hline 15 & 605 & 542 & 510 & úfortócetirE \\
\hline 0 & 634 & 661 & 1020 & 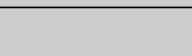 \\
\hline 0 & 634 & 661 & 1360 & 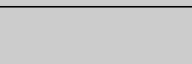 \\
\hline
\end{tabular}

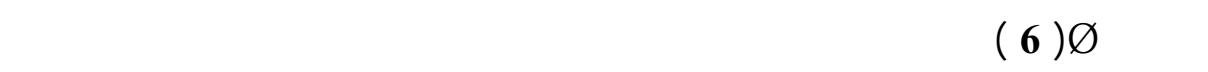

\begin{tabular}{|c|c|c|c|c|}
\hline 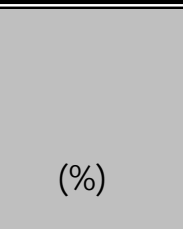 & 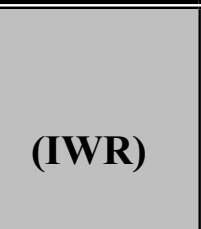 & 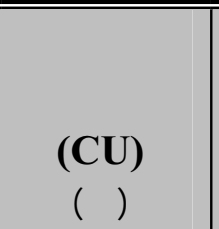 & 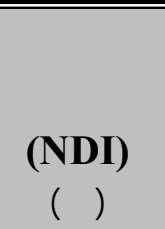 & 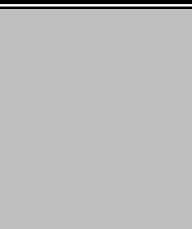 \\
\hline 23 & 645 & 522 & 480 & úfouttéet|rE \\
\hline 3.7 & 671 & 667 & 960 & 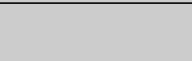 \\
\hline 2.5 & 679 & 675 & 1280 & 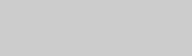 \\
\hline
\end{tabular}




\section{tt'요지}

1- Cavero, Jase, Inma Farre, Philippe Debaeke, and Jose, M. Faci. (2000). Simulation of maize yield under water stress with the EPICphase and CROPWAT models. Journal of Agronomy, Vol.92, No.4: 679 -690.

2- FAO, (1992). CROPWAT, a computer programe for irrigation planning and management. Irrigation and Drainage Paper No.42, Rome, United Nations.

3- FAO, (1998). Crop evapotranspiration guidelines for computing cropwater requirements. Irrigation and Drainage Paper No.56, Rome, United Nations.

4- Martin, S.O., Fabiro, C., Ramos, A.B. and Serra, A.L. (1999). Irrigation scheduling. In: Vanlier, H.N. ed., CIGR Hand Book of Agriculture, Vol.I (Land and Water Engineering), CIGR .

5- Snyder ,R.L, Stephen, R. G., and Larry J . S.(2002). Drought tips for vegetable and field crop production. University of California Division of Agriculture and Natural Resources.

6- Sumner, D.M. (1996). Evapotranspiration from successional vegetation in a deforested area of the Lake Wales Ridge. Report No. 96:4244. University of Florida, USA.

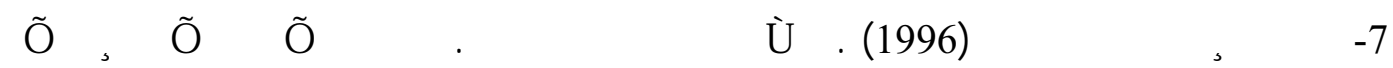

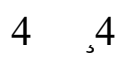

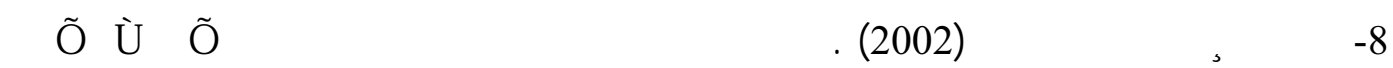

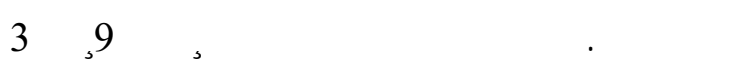

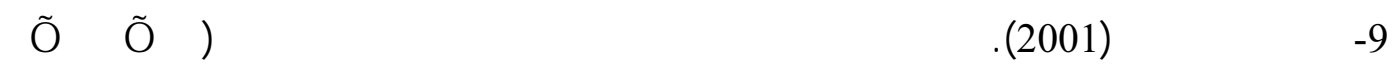
.(น̃åđ 